\title{
CRECIMIENTO ECONÓMICO, GOBERNABILIDAD DEMOCRÁTICA Y DESARROLLO SOCIAL: UN ENFOQUE INTEGRADOR
}

\author{
Joaquín Guzmán Cuevas* \\ Isidoro Romero Luna**
}

Fecha de recepción: 16 de noviembre de 2007. Fecha de aceptación: 4 de marzo de 2008.

\section{Resumen}

En este trabajo se plantea una caracterización de los procesos de desenvolvimiento socioeconómico sustentada en la hipótesis de existencia de circularidades o procesos de causalidad acumulativa que impulsan hacia adelante a los países hiperdesarrollados y atrapan a los hipodesarrollados, condenándolos a situaciones de atraso relativo. Asimismo, en el marco de esta visión circular del desarrollo se discute la significación de la gobernabilidad democrática en los procesos de desarrollo en dos niveles: desde la perspectiva interna de los propios países hipodesarrollados afectados por situaciones de inestabilidad política o sometidos a regímenes autoritarios, y desde la visión de la gobernabilidad a escala global en el actual escenario de globalización dominado por las fuerzas del mercado.

Palabras clave: desarrollo, gobernabilidad, democracia, círculos viciosos y círculos virtuosos.

* Catedrático de Economía Aplicada en la Universidad de Sevilla, España. Correo electrónico:jjguzman@us.es.

** Catedrático en Economía por la Universidad de Sevilla, España. Correo electrónico: isidoro@us.es. 


\section{Summary}

This study presents a characterization of the unfolding socio-economic processes sustained by the hypothesis of the existence of circularities or accumulative causality processes that drive forward the hyper-developed countries and trap the hypodeveloped countries, condemning the latter to situations of relative backwardness. At the same time, within the framework of this circular vision of development, we discuss the meaning of democratic governance in development processes at two levels: from the internal perspective of the hypo-developed countries themselves affected by situations of political instability or subjected to authoritarian regimes, and from the point of view of governance at a global scale in the current scenario of globalization dominated by market forces.

Key words: development, governance, democracy, vicious circles and virtuous circles.

\section{Résumé}

Dans ce travail est proposée une caractérisation des processus de développement socio-économique fondée sur l'hypothèse de l'existence de circularités ou processus de causalité accumulative qui poussent en avant les pays hyper-développés et bloquent les pays hypo-développés, en les condamnant à des situations de retard relatif. Ainsi, dans le cadre de cette vision circulaire du développement, est discuté le sens de la gouvernabilité démocratique dans les processus de développement sur deux plans: depuis la perspective interne des pays hipo-développés eux-mêmes affectés par des situations d'instabilité politique ou soumis à des régimes autoritaires, et sous l'angle de la gouvernabilité à l'échelle mondiale dans le contexte actuel de mondialisation dominée par les puissances du marché.

Mots clés: développement, gouvernabilité, démocratie, cercles vicieux et cercles virtueux.

\section{Resumo}

Neste trabalho se propõe uma caracterização dos processos de desenvolvimento socioeconômico sustentada na hipótese de existência de circularidades ou processos de causalidade acumulativa que impulsionam para frente os países hiperdesenvolvidos e amarram os países hipodesenvolvidos, condenando-os a situações de atraso relativo. Ao mesmo tempo, no marco desta visão circular do desenvolvimento discute-se a significação da governabilidade democrática nos processos de desenvolvimento en dois níveis: desde a perspectiva interna dos próprios países hipodesenvolvidos afetados por situações de inestabilidade política ou submetidos a regimes autoritários, e do ponto de vista da governabilidade a escala global no atual cenário de globalização dominado pelas forças do mercado.

Palavras-chave: desenvolvimento, governabilidade, democracia, círculos viciosos e círculos virtuosos. 


\section{Introducción}

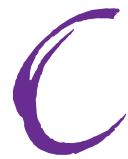

ualquier aproximación al complejo problema del desarrollo debe partir de dos aspectos esenciales que lo caracterizan como fenómeno social: su carácter autosostenido y su naturaleza multidimensional. Por una parte, la polarización de la riqueza a escala mundial incita a considerar la existencia de mecanismos de autosostenimiento o retroalimentación generadores de inercias que, en el caso de los países más desarrollados, impulsan los procesos de avance, mientras que en el caso de las naciones más atrasadas, obstaculizan las posibilidades de progreso. Por otra parte, el fenómeno del desarrollo tiene una naturaleza intrínsecamente multidimensional, tanto cuando se observa desde una perspectiva positivo-descriptiva, como cuando se analiza desde una óptica normativo-teleológica. Desde una perspectiva positivodescriptiva, el proceso de desarrollo no puede entenderse plenamente si se consideran exclusivamente variables económicas, sin atender a factores de carácter sociológico, cultural y político; desde un punto de vista teleológico; la idea de progreso implícita en la noción de desarrollo no sólo comprende mejoras en el plano económico, sino también transformaciones en las dimensiones social y político-institucional, que resultan imprescindibles para hablar de auténtico desarrollo.

A partir de estos principios y de las ideas introducidas por Gunnar Myrdal (1974) o Ragnar Nurkse (1955) entre otros, en Fontela y Guzmán (2003 y 2006) se plantea una visión de los procesos de desenvolvimiento socioeconómico sustentada sobre la existencia de circularidades o procesos de causalidad acumulativa que impulsan hacia adelante a los países hiperdesarrollados y atrapan a los hipodesarrollados, condenándolos a situaciones de atraso relativo. No obstante, los círculos viciosos y virtuosos allí contemplados son producto exclusivamente de la dinámica interna de las naciones hipo e hiperdesarrolladas, sin considerar explícitamente el efecto que su inserción en el orden internacional puede tener sobre sus dinámicas internas. Sin embargo, en el marco actual de globalización, no cabe duda de que existen intensos vínculos de interdependencia asimétrica que enlazan las estructuras socioeconómicas de los países hiperdesarrollados e hipodesarrollados. Por tanto, el análisis de la problemática del desarrollo no puede limitarse a la consideración de variables internas, sino que resulta imprescindible incorporar el impacto de factores externos en la dinámica interna de las naciones.

Así pues, en el presente trabajo, a partir de las ideas propuestas en Fontela y Guzmán (2003 y 2006), se plantea una versión más general de la teoría circular del desarrollo que incorpora los impactos externos sobre las dinámicas circulares internas. Este marco de referencia global permite plantear hipótesis contrapuestas: desde la 
eventual existencia de un círculo internacional de la pobreza que sumaría sus efectos a los círculos viciosos internos de los países hipodesarrollados, hasta la inserción de las naciones hipodesarrolladas en el orden internacional como vía de ruptura de los círculos viciosos internos del hipodesarrollo.

Por otra parte, en los últimos tiempos se viene prestando una atención creciente al impacto que la organización política y el funcionamiento de las instituciones de gobierno tienen sobre el proceso de desarrollo (véase, por ejemplo, Barro, 1994; Helliwell, 1994; Feng, 1997; Rodrik, 2000; Tavares y Wacziarg, 2001; Kaufmann y Kraay, 2002; PNUD, 2002; Kaufmann, Kraay y Zodio-Lobatón, 2003). En este trabajo, desde el marco interpretativo que representa la teoría circular del desarrollo, también se analiza la significación de la gobernabilidad democrática en el proceso de desarrollo en dos niveles: desde la perspectiva interna de los propios países hipodesarrollados, afectados por situaciones de inestabilidad política o sometidos a regímenes autoritarios, y desde la visión de la gobernabilidad a escala global en el actual escenario de globalización dominado por las fuerzas del mercado.

\section{Una versión compleja de la teoría circular del desarrollo La dinámica interna del hiper e hipodesarrollo}

Fontela y Guzmán (2003 y 2006) plantean una aproximación abstracta y holística del proceso de desarrollo a partir de un sistema conformado por las relaciones de interdependencia entre variables y fenómenos de carácter económico, sociológico y político; la estructura causal de este sistema podría expresarse en forma de una matriz cuyos elementos recogerían los efectos de unas variables o fenómenos sobre los otros. ${ }^{1}$ De este modo, cabe plantear una visión compleja y multidimensional de los círculos viciosos internos en los que se ven atrapados los países hipodesarrollados, y de los círculos virtuosos de los que se beneficiarían los países hipodesarrollados tal como sigue:

En la esfera económica, los países hipodesarrollados (hiperdesarrollados) acreditan un débil (fuerte) esfuerzo inversor que condiciona el estancamiento (la expansión) del producto y de la renta nacional. A su vez, el bajo (alto) nivel de renta limita (aumenta) la capacidad de ahorro y, por tanto, la disponibilidad de recursos para financiar la inversión productiva, cerrándose así un círculo vicioso (virtuoso) de naturaleza económica.

1 En Guzmán y Fontela (2003) se plantea una matriz binaria $A$, donde los elementos $a_{i j}=1$, si $j$ es causa de $i$ y $a_{i j}=0$, en caso contrario, o alternativamente una matriz $A$ donde $a_{i j}=+$, si el efecto de la acción $j$ es positivo para $i ; a_{i j}=0$, si el efecto es nulo o $a_{i j}=-$, si el efecto de la acción $j$ es negativo para $i$. 
Por otra parte, en el plano social, se pueden identificar igualmente círculos endógenos de causalidad acumulativa según el siguiente esquema:

Las economías hipodesarrolladas (hiperdesarrolladas) se caracterizan por un sistema educativo muy deficiente (sólido), que impide (favorece) el pleno desarrollo de las capacidades de la población y transmite pautas escasamente (altamente) racionales obstaculizadoras (impulsoras) del progreso social. En el caso de los países hipodesarrollados, se mantienen así prejuicios y actitudes regresivas propiciadoras de un contexto conformista y escasamente crítico, que condiciona un cierto desinterés por la educación. Por consiguiente, se manifiesta también en este ámbito social un círculo vicioso de causalidad acumulativa que limita el desarrollo humano en los países más atrasados. Por el contrario, en las naciones hiperdesarrolladas las actitudes y valores progresivos generan un proceso de avance social y mayores niveles de conocimientos científicos y tecnológicos, que refuerzan a su vez el sistema educativo.

En tercer lugar, con respecto a la dimensión política, cabría identificar procesos endógenos de causalidad acumulativa a partir del siguiente esquema:

Los países hipodesarrollados (hiperdesarrollados) suelen caracterizarse por formas de gobierno dictatoriales o autocráticas (democráticas), que limitan (fortalecen) las libertades civiles y la participación de los ciudadanos en la vida pública. En las naciones hipodesarrolladas la restricción de las libertades y la ausencia de participación democrática se traducen en falta de transparencia y corrupción. De este modo, se fragua un marco de inestabilidad política e ingobernabilidad que constituye el caldo de cultivo idóneo para la imposición por la fuerza de regímenes autoritarios. Se completa, consecuentemente, un círculo vicioso conformado, en este caso, por relaciones de causalidad en el ámbito político. Por el contrario, en los países hiperdesarrollados, el funcionamiento transparente y eficaz de las instituciones favorece el mantenimiento de una estabilidad sociopolítica básica necesaria para la consolidación de un régimen democrático.

Finalmente, la dinámica interna del hipo y del hiperdesarrollo se caracteriza por la conexión central que conforma un circuito integrador de interdependencia económica-política-social. De este modo, las relaciones entre los tres subsistemas económico, político y social por la vía de las relaciones entre la inversión, la estabilidad y la educación establecen unas conexiones - de carácter positivo, en el caso del hiperdesarrollo y de carácter negativo, en el caso del hipodesarrollo- entre los tres círculos viciosos o virtuosos descritos anteriormente, como se recoge en la figura 1 para el caso del hiperdesarrollo. 
Figura 1

Círculos virtuosos del hiperdesarrollo

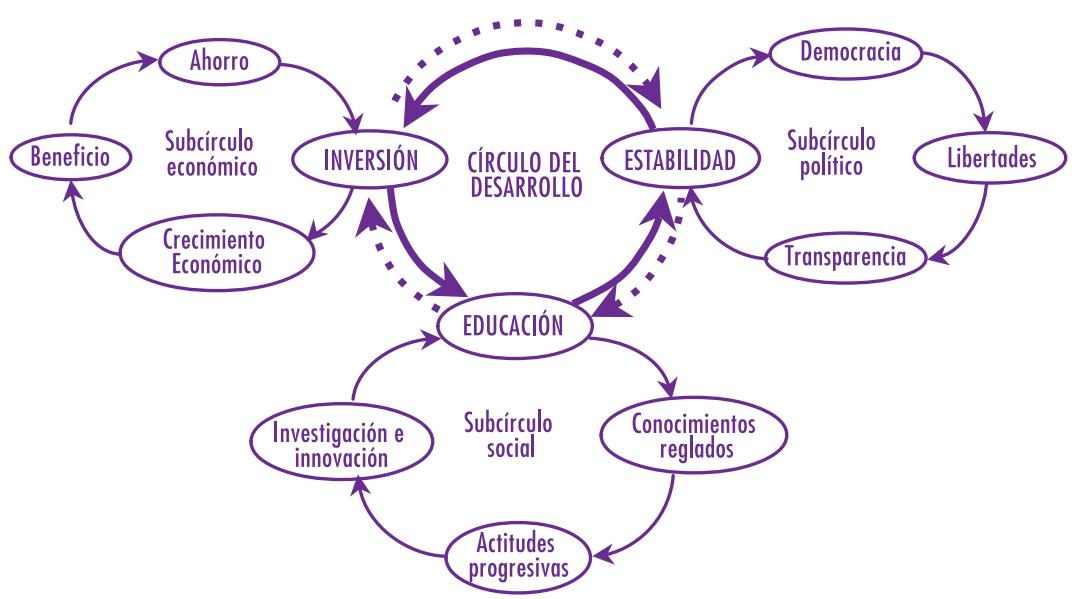

\section{Los factores externos en la teoría} circular del desarrollo

La versión anterior de la teoría circular del desarrollo sólo considera las dinámicas internas de los países hipo e hiperdesarrollados, sin que se analice el efecto de la inserción de esos procesos en un orden internacional caracterizado por una interdependencia creciente. Por el contrario, adoptamos aquí una visión global a partir del conjunto del sistema mundial. Desde esta perspectiva, la matriz de estructura causal para el sistema de interrelaciones (figura 2) podría dividirse en cuatro submatrices que reflejarían cuatro modelos explicativos parciales:

Figura 2

Teoría circular del desarrollo

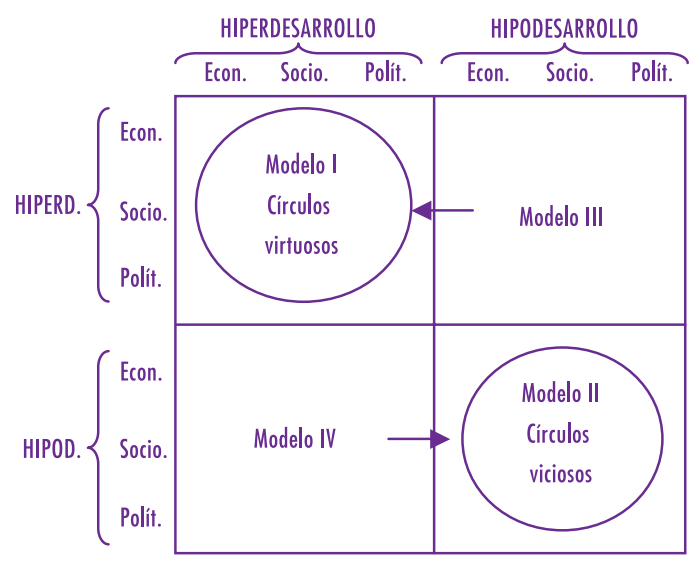


a) El Modelo I recogería las relaciones entre los factores de carácter económico, social y político en los países más avanzados, caracterizando la dinámica interna del subsistema del hiperdesarrollo. Este modelo interpretativo respondería al esquema de los círculos virtuosos del hiperdesarrollo planteados en Guzmán y Fontela (2003 y 2006).

b) El Modelo II recogería las relaciones entre los factores de carácter económico, social y político en los países más atrasados, caracterizando la dinámica interna del hipodesarrollo. Este modelo interpretativo reflejaría la existencia de los círculos viciosos del hipodesarrollo planteados en Guzmán y Fontela (2003 y 2006).

c) El Modelo III recogería la repercusión de las relaciones internacionales con los países del hipodesarrollo sobre los procesos económicos, sociales y políticos de las naciones del hiperdesarrollo. Este modelo informaría sobre la naturaleza del impacto exógeno de los países hipodesarrollados sobre los círculos virtuosos endógenos de los que se benefician las naciones hiperdesarrolladas.

d) El Modelo IV recogería la influencia sobre los procesos económicos, sociales y políticos de los países del hipodesarrollo derivada de sus relaciones con las naciones hiperdesarrolladas. Este modelo nos informaría sobre el impacto exógeno de los países hiperdesarrollados sobre los círculos viciosos endógenos que conducen al estancamiento de las naciones del hipodesarrollo.

Los modelos III y IV se conforman por múltiples relaciones internacionales de carácter económico, sociocultural y político que pueden clasificarse, para ambos casos, en dos grupos:

\section{Figura 3}

Factores externos de aceleración (izq.) y desaceleración (der.)
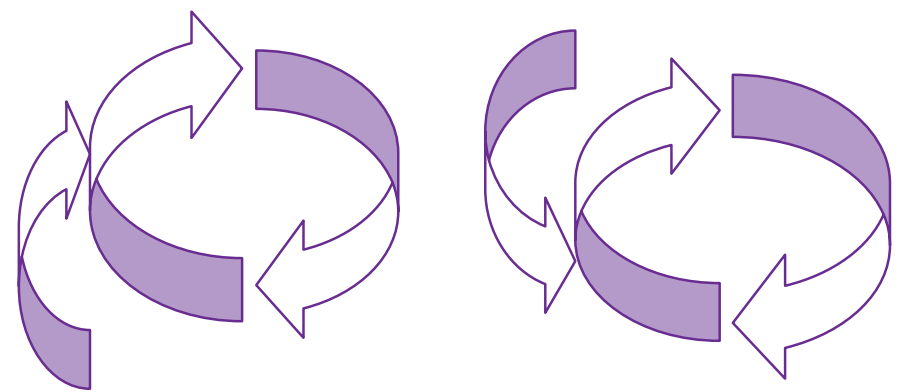

- Los vínculos o relaciones entre factores que refuerzan los procesos internos de causalidad circular, al actuar en el mismo sentido de la inercia original. Se trata, 
por tanto, de aquellas relaciones internacionales que contribuyen a la retroalimentación de los círculos virtuosos del hiperdesarrollo o de los círculos viciosos del hipodesarrollo. A estas relaciones de causalidad las denominamos factores externos de aceleración de la dinámica circular del desarrollo.

- Los vínculos que actúan en el sentido contrario a las inercias internas en la dinámica de causalidad circular. Se trata, en este segundo caso, de las relaciones externas que podrían desencadenar desde fuera la "ruptura" de la dinámica "virtuosa" del hiperdesarrollo o de la dinámica "viciosa" del hipodesarrollo. A estas relaciones de causalidad las denominamos factores externos de desaceleración de la dinámica circular del desarrollo.

A continuación, de modo exploratorio y sin ánimo de exhaustividad, se identifican algunos de los impactos exógenos adscritos al Modelo IV que describe el funcionamiento de los círculos viciosos de hipodesarrollo en el marco de una economía abierta.

Las relaciones internacionales repercuten sobre los círculos viciosos del hipodesarrollo de diverso modo: algunos factores externos aceleran la dinámica circular de la pobreza, mientras que otros impactos externos actúan en el sentido contrario a la inercia de estancamiento interno.

\section{Factores de aceleración}

Los países del hipodesarrollo pueden ver reforzados sus círculos viciosos internos de la pobreza como consecuencia de sus relaciones con las naciones más avanzadas por diversas vías:

- Respecto al subcírculo vicioso económico. Por un lado, la competencia de los productos y servicios procedentes de los países del hiperdesarrollo aboca a las naciones hipodesarrolladas a un patrón de especialización poco favorable, centrado en productos básicos y productos industriales de reducido valor añadido y nivel tecnológico bajo, intensivos en recursos naturales o mano de obra. ${ }^{2}$ Asimismo, la fuga de capitales imputable a las élites dominantes en los países del hipodesarrollo, que colocan su ahorro en los sistemas financieros de las naciones más avanzadas, evita que estos recursos sean canalizados por los sistemas financieros locales hacia los procesos de inversión internos. De modo semejante, la retribución del ahorro ex-

2 En cualquier caso, es necesario puntualizar que la presencia de las importaciones en la figura 4 alude exclusivamente a su repercusión directa sobre el flujo circular de la renta a escala nacional. No obstante, cabría también señalar el impacto favorable de esta variable en la oferta y su contribución positiva e irremplazable en cualquier economía abierta. 
terno constituye otra vía de escape — en forma de dividendos, intereses, etcéteraen el ciclo interno de ahorro-inversión. En este punto, cabe resaltar especialmente el costo insufrible que supone la deuda externa para muchas naciones atrasadas en las que alcanza niveles insostenibles. Entre otros efectos negativos, el esfuerzo derivado del servicio de la deuda limita los recursos disponibles por el Estado para inversiones en capital físico y humano (educación, sanidad, etcétera).

Figura 4

Factores externos de aceleración del círculo vicioso del hipodesarrollo

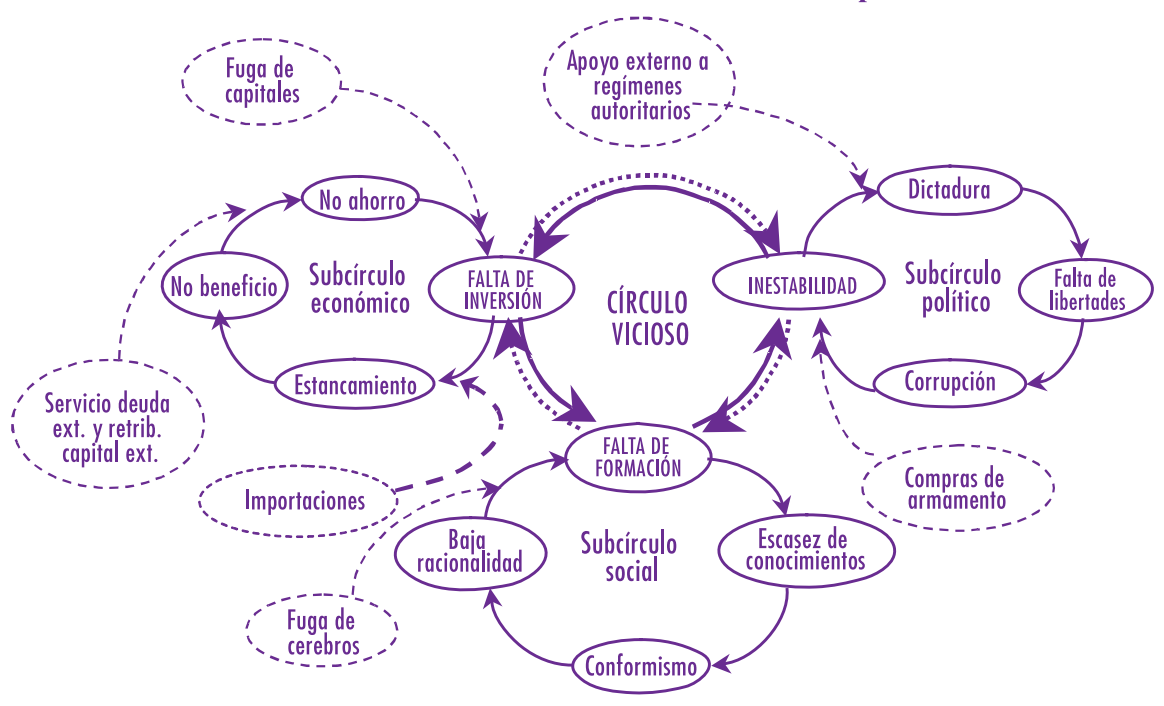

- Respecto al subcírculo vicioso social. En el ámbito social, hay que tener presente el impacto negativo derivado de la fuga de cerebros, que supone una pérdida de capital humano para las zonas de origen.

- Respecto al subcírculo vicioso político. En algunos casos la política exterior de los países más avanzados, orientada por intereses estratégicos nacionales, ha actuado en contra de procesos de democratización en naciones atrasadas al apoyar el establecimiento o contribuir a consolidar regímenes autoritarios. Asimismo, la industria armamentística constituye un sector potente en las economías más avanzadas que se nutre en buena medida de los conflictos bélicos locales en países pobres. El negocio armamentístico vincula los intereses económicos de grandes empresas y gobiernos de las naciones hiperdesarrolladas con la inestabilidad política y la guerra en los países más pobres. 


\section{Factores de desaceleración}

Sin embargo, los naciones del hipodesarrollo también obtienen beneficios de sus relaciones con los países hiperdesarrollados, actuando su inserción en la economía internacional como una fuerza contraria a la inercia de estancamiento prevaleciente.

- Respecto al subcírculo vicioso económico. Las exportaciones de bienes y servicios hacia los países del hiperdesarrollo, pese a la reducida capacidad exportadora de las naciones hipodesarrolladas y a su débil acceso a los mercados de las economías más potentes, contribuyen al crecimiento económico de los países más pobres. Asimismo, el ahorro externo representa una vía de financiación de los procesos de inversión de las naciones del hipodesarrollo. Las economías más atrasadas pueden superar las restricciones financieras a sus procesos de formación de capital mediante su acceso a los mercados financieros internacionales. De esta forma, estos países pueden romper el círculo vicioso económico determinado por sus bajos niveles de ingreso, su baja tasa de ahorro interno y su reducida inversión, escapando así de situaciones de equilibrio de bajo nivel. A este respecto, consideramos aquí tanto la inversión extranjera directa como cualquier otra vía de financiación externa. De modo semejante, la ayuda al desarrollo, pese a que su eficacia y eficiencia están sujetas a muchas interrogantes (véase, por ejemplo, Larrú, 2003 y Alcaide y Larrú,

Figura 5

Factores externos de desaceleración del círculo vicioso del hipodesarrollo

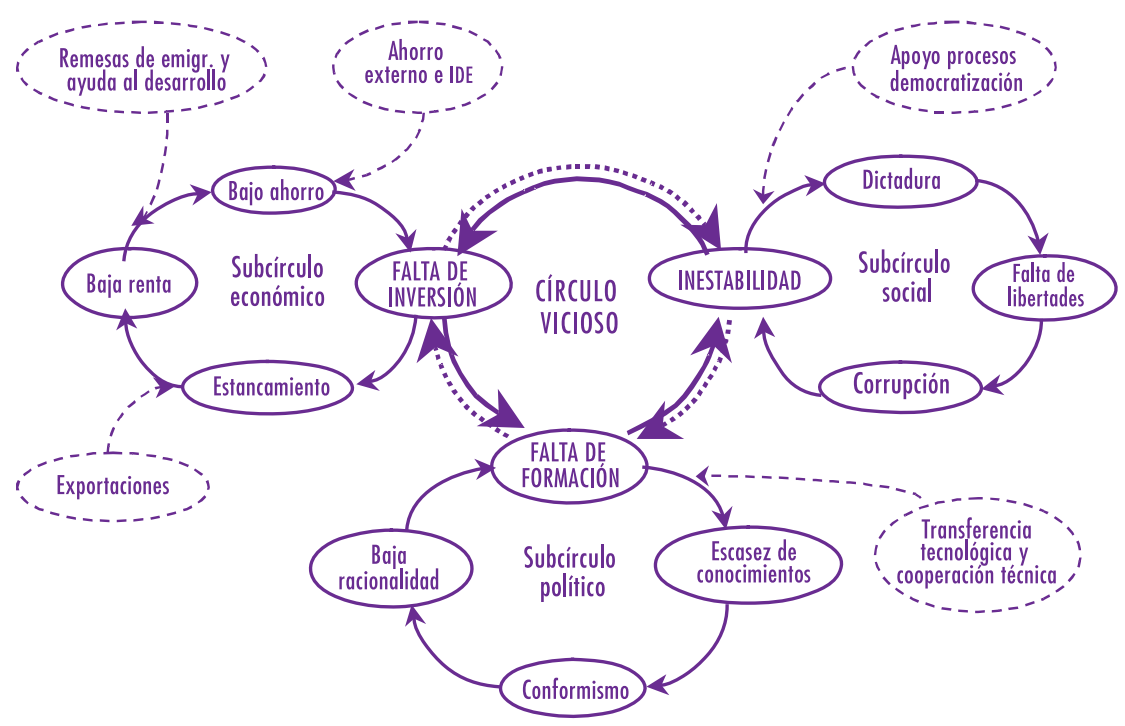


2007), tiene un impacto positivo sobre los países más necesitados, al igual que las remesas de los emigrantes que consiguen empleo en las áreas más desarrolladas.

- Respecto al subcírculo vicioso social. La transferencia tecnológica y la asistencia técnica constituyen vías de acceso a la tecnología desarrollada en el exterior, permitiendo la elevación de los niveles de productividad de las economías más atrasadas sin requerir procesos de innovación propios.

- Respecto al subcírculo vicioso político. El apoyo a los procesos de democratización de la comunidad internacional puede favorecer el cambio político interno mediante la presión diplomática y la cooperación técnica.

\section{El papel de la gobernabilidad en los círculos viciosos y virtuosos del desarrollo}

En la exposición anterior se ha planteado una aproximación al desarrollo sobre la base de un enfoque integrador de las dimensiones económica, social y política que lo configuran. De esta forma, el desarrollo aparece conceptuado como un proceso de acumulación de capital en una triple acepción:

- Acumulación de capital físico o productivo en el ámbito económico.

- Acumulación de capital humano y social en el ámbito social.

- Acumulación de capital institucional en el ámbito político.

Más específicamente, en el plano político, el proceso de conformación de las instituciones, las reglas de juego y los cauces operativos de gobierno aceptados por la colectividad — que se ha plasmado en el subcírculo político — representa en última instancia un proceso de obtención de gobernabilidad. A este respecto, entendemos, siguiendo a Prats (2001), que un sistema social es gobernable "cuando está estructurado sociopolíticamente de modo que todos los actores estratégicos se interrelacionan para tomar decisiones colectivas y resolver los conflictos conforme a un sistema de reglas $\mathrm{y}$ de procedimientos formales e informales — que pueden registrar diversos niveles de institucionalización - dentro del cual formulan sus expectativas y sus estrategias".

La idea de gobernabilidad no implica en sí misma la existencia de reglas de juego democrático; sino que, por el contrario, es aplicable al caso de dictaduras y regímenes autoritarios de todo tipo, en tanto sólo requiere la existencia de instituciones que procuren un cierto orden social y una estabilidad política suficiente, aunque este orden venga impuesto por la fuerza (Prats, 2001). Sin embargo, como defiende 
Amartya Sen (1998), el desarrollo está estrechamente ligado a la democracia y a las libertades, constituyendo la pobreza en definitiva - tanto en el plano personal como colectivo - una situación de ausencia de libertad. Por tanto, el modo de gobernabilidad que debemos considerar como auténtica sustancia del desarrollo es la gobernabilidad democrática.

\section{Gobernabilidad democrática, crecimiento}

económico y desarrollo social

En el modelo propuesto en la primera parte de este trabajo se encuentran incorporadas las relaciones entre la gobernabilidad democrática y el crecimiento económico, por un lado, y entre la gobernabilidad democrática y el desarrollo social — vinculado a la elevación de los niveles educativos y una cierta equidad social—, por otro. No obstante, a continuación trataremos de profundizar algo más en el análisis de estas relaciones (figura 6).

Las relaciones entre gobernabilidad, democracia y crecimiento económico

Para considerar específicamente el papel de la gobernabilidad democrática en la dinámica circular del desarrollo conviene tomar en cuenta por separado los dos elementos que la conforman: la gobernabilidad, entendida como simple estabilidad política, y la democracia. ${ }^{3}$

\section{Figura 6}

El papel de la gobernabilidad democrática en el proceso de desarrollo

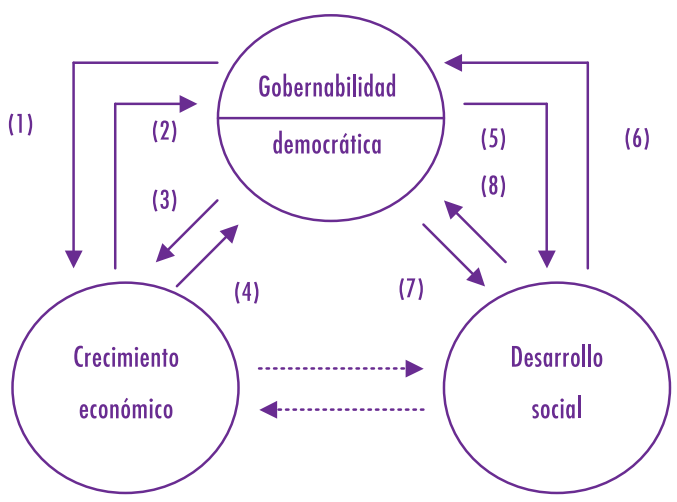

3 Al seguir a Dahl (1998), aquí se asume que la democracia implica de modo general: participación efectiva; igualdad de voto; posibilidad de entendimiento informado y ejercicio de control final sobre la agenda y la inclusión de asuntos. 
La relación gobernabilidad-crecimiento económico está siendo objeto de gran atención en los últimos tiempos (Kaufmann y Kraay, 2002; Kaufmann, Kraay y Zoido-Lobatón, 2002). A este respecto, el análisis se ha referido fundamentalmente al impacto de la estabilidad política como factor impulsor del crecimiento (impacto 1 en figura 6). Desde esta perspectiva, como se ha señalado con anterioridad, una cierta paz social representa un factor trascendental para eliminar las incertidumbres que frenan los procesos de inversión. Ciertamente, el correcto funcionamiento de los mecanismos de mercado requiere de un adecuado desenvolvimiento del proceso político, sin que existan fricciones que amenacen la estabilidad básica del sistema. En concreto, la continuidad del ciclo ahorro-inversión-ahorro requiere de un marco institucional que preserve los derechos de propiedad, la libertad de empresa y la obligatoriedad del cumplimiento de los contratos. Estas condiciones se favorecen cuando existe un clima de paz social sin conflictos que amenacen la permanencia de las reglas y procedimientos que regulan el acceso y el ejercicio del poder. A este respecto, Feng (1997), entre otros, encuentra evidencia empírica del impacto negativo de la inestabilidad política — entendida como cambios de régimen político— sobre el crecimiento.

En cualquier caso, es necesario tener presente que aunque la mera gobernabilidad - entendida en los términos anteriores - resulta favorecedora del crecimiento económico, debemos referir el análisis al concepto más amplio y rico de desarrollo, lo que conduce, como hemos señalado, a la idea de gobernabilidad democrática. Así pues, mientras que la gobernabilidad puede considerarse como un medio para impulsar el crecimiento y el desarrollo, la gobernabilidad democrática es a la vez un medio y un objetivo de desarrollo.

En este sentido, algunos autores han considerado la incidencia de la democracia sobre los procesos económicos y más específicamente en el crecimiento (Helliwell, 1994; Feng, 1997; Rodrik, 2000) (impacto 3). En línea general, a la luz de los estudios realizados, no parece que haya un fuerte efecto directo de la democracia sobre el crecimiento económico. Sin embargo, Rodrik (2000) comprueba que las democracias se comportan mejor que los regímenes autoritarios en cuatro aspectos: conducen a tasas de crecimiento a largo plazo más predecibles, garantizan mayores niveles de estabilidad en el comportamiento económico, se enfrentan mejor a las crisis económicas y proporcionan niveles salariales superiores. No obstante, el eventual impacto de la democracia sobre el crecimiento se produce, a juicio de algunas opiniones, de un modo indirecto por medio del efecto positivo de la democracia sobre los niveles educativos o sobre la estabilidad del sistema político (Helliwell, 1994; Feng, 1997). 
Por su parte, Barro (1994) encuentra una relación no lineal entre la democracia y el crecimiento, de modo que en situaciones de bajos niveles de libertades políticas el crecimiento se elevaría ante mayores cotas de democracia; sin embargo, en sociedades que ya han alcanzado ciertos niveles de democracia, el crecimiento se vería afectado de manera negativa por las mejoras democráticas. Finalmente, algunos autores han planteado la hipótesis de existencia de un conflicto entre derechos políticos y rendimiento económico. En este sentido, Tavares y Wacziarg (2001) aprecian un cierto efecto global negativo de la democracia sobre el crecimiento, que, consideran, se debe a un impacto negativo de la democracia sobre la acumulación de capital físico.

Por otro lado, habría que tomar en cuenta el posible impacto del crecimiento económico sobre la gobernabilidad democrática (impactos 2 y 4 en la figura 6). En una primera aproximación, podríamos asumir como hipótesis plausible que el crecimiento económico, en la medida en que se traduce en una mejora del bienestar general de la población, favorece la estabilidad política al limitar el surgimiento de tensiones sociales (impacto 2 en figura 6).

En esta línea, en las consideraciones realizadas en los apartados anteriores se ha defendido la posible existencia de un círculo virtuoso crecimiento-gobernabilidad democrática-crecimiento. Sin embargo, Kaufmann y Kraay (2002) opinan que el crecimiento no tiene por qué redundar automáticamente en una mejora de la gobernabilidad, entendida en sentido amplio y como gobernabilidad democrática (impactos 2 y 4). ${ }^{4}$ De esta manera, no se desencadenarían automáticamente procesos de causalidad circular acumulativa o círculos virtuosos que propulsen en paralelo el crecimiento económico y la gobernabilidad democrática. En su opinión, este resultado se explica, entre otros factores, por la eventualidad de que las élites dominantes en países atrasados se estén beneficiando de un deficiente desarrollo de las instituciones y de la ausencia de gobernabilidad, por lo que se resistirían a cualquier proceso de fortalecimiento institucional y refuerzo de los cauces democráticos. De este modo, se da lugar a un fenómeno de captura del Estado, entendido como la influencia ilegítima de las élites en el proceso de establecimiento de la legislación y de las políticas gubernamentales (Kaufmann y Kraay, 2002). Como ejemplo de este

4 Kaufmann y Kraay (2002) parten de una definición de gobernabilidad que incluye el proceso de elección de los gobiernos, su capacidad de formular y aplicar efectivamente las políticas y el respeto de los ciudadanos y del Estado a las instituciones que regulan las interacciones económicas y sociales. 
tipo de situaciones los autores señalan los casos de algunos nuevos países industrializados del sureste asiático o de algunas naciones latinoamericanas.

No obstante, a nuestro juicio y desde la perspectiva holística que adoptamos, la falta de repercusión del crecimiento económico sobre la gobernabilidad vendría dada realmente, no tanto por la inexistencia de un efecto directo, sino por un bloqueo en el ámbito del círculo social. Ciertamente, cuando los beneficios del crecimiento no se reparten en términos suficientemente equitativos y redundan en forma de mejoras educativas, sanitarias y en las condiciones de vida de la generalidad de la población, se deterioran las bases sociales que requiere el funcionamiento adecuado del proceso político. De este modo, se frena la generación de una inercia virtuosa de desarrollo como consecuencia de la parálisis en el engranaje social.

En cualquier caso, y refiriéndonos específicamente al impacto del crecimiento y del nivel de renta sobre la democracia (4 en la figura 6), se ha apreciado en diversos trabajos una repercusión favorable (Helliwell, 1994; Barro, 1999).

Gobernabilidad, democracia y desarrollo social

Por otra parte, en lo que atañe a la relación gobernabilidad democrática-desarrollo social, como se señalara con anterioridad en el segundo apartado, el esfuerzo formativo fundamental en el desarrollo de una comunidad representa, en última instancia, un proceso de inversión que requiere de la existencia de un clima de seguridad y tranquilidad. Desde este punto de vista, la gobernabilidad y la estabilidad política facilitarán el correcto desenvolvimiento del sistema educativo y la formación de capital humano (impacto 5). Asimismo, en sentido inverso, los mayores niveles educativos pueden reflejarse en actitudes de mayor responsabilidad social y tolerancia que favorecen la estabilidad sociopolítica (impacto 6).

En particular, cuando la conformación del poder político emana de un proceso democrático, cabe esperar de las instituciones una mayor atención para la educación, de modo tal que, desde esta perspectiva, la democracia incidiría positivamente sobre el nivel formativo de la población (impacto 7). Esta hipótesis ha sido contrastada favorablemente, entre otros, por Helliwell (1994).

Asimismo, en el sentido inverso, la consolidación de un régimen democrático requiere de un nivel educativo elevado y de una cultura política de la democracia ampliamente extendida en la población (impacto 8). A este respecto, hay que tener presente que la democracia no viene dada inmediatamente por la introducción de las instituciones democráticas formales (elecciones libres, sufragio universal, libertad de asociación política, etcétera), sino que precisa de una actitud proactiva de la po- 
blación interesada en el proceso político. Una democracia sin participación es una democracia vacía. Por tanto, la profundización democrática pasa por la existencia de una población altamente formada y preocupada por los asuntos públicos que haya asumido plenamente los valores democráticos. Sin el logro de estándares mínimos en educación y equidad social no puede funcionar una auténtica democracia, todo lo más que se alcanzará serán seudodemocracias que incorporan algunos elementos mínimos de democracia electoral, pero sometidos en última instancia a mecanismos de control autoritario por parte de oligarquías o liderazgos personalistas. En este sentido, Barro (1999) obtiene evidencia favorable al impacto positivo de la educación primaria sobre la democracia, medida por un indicador subjetivo de derechos electorales.

\section{La gobernabilidad democrática y el orden internacional}

El modelo propuesto en la primera parte de este trabajo considera explícitamente la influencia de los factores externos sobre la dinámica circular del desarrollo. Ciertamente, en la etapa actual de la historia de la humanidad la dimensión global de los procesos económicos, sociales y políticos cobra más importancia de manera progresiva, frente a los condicionamientos y fenómenos a escala nacional o regional. Por tanto, cualquier estrategia global orientada al desarrollo de las zonas más desfavorecidas, por medio de la ruptura de los círculos viciosos que afectan a los países más pobres, debe tener presente los impactos que desde el exterior se ocasionan sobre los procesos sociopolíticos en las naciones hipodesarrolladas.

A este respecto, hay que considerar las repercusiones, en muchos casos desfavorables, de la política exterior de los países más avanzados sobre la situación sociopolítica interna de las naciones hipodesarrolladas. En este sentido, debería reforzarse desde el exterior el apoyo a los procesos de democratización o de consolidación democrática y nunca fortalecer modelos políticos que garanticen una cierta gobernabilidad sobre bases no democráticas, circunstancia de la que no es difícil encontrar ejemplos significativos. Por el contrario, ante situaciones de estabilidad política en condiciones no democráticas la comunidad internacional debe apoyar a los actores estratégicos emergentes que generen un conflicto interno al promover cambios en la institucionalidad existente. En estas situaciones conviene contribuir a la alteración de las condiciones de la gobernabilidad vigente a fin de alcanzar un cambio político del que resulte un modelo gobernable sobre principios democráticos (Prats, 2001). Todo ello desde la convicción profunda de que la democracia no puede imponerse ni introducirse artificialmente "desde afuera", sino que la construcción democrática 
debe ser esencialmente un proceso endógeno impulsado por los esfuerzos y anhelos de la población.

Por otra parte, en la actualidad asistimos a una clara contradicción entre un orden económico altamente globalizado frente a la ausencia de instituciones de ámbito mundial con auténtica capacidad operativa para incidir sobre los problemas globales (subdesarrolllo, pobreza, problemas ambientales, terrorismo internacional, inestabilidad política y conflictos armados, etcétera). A ello se une el que los antiguos Estadosnación se muestran desbordados por la naturaleza de un proceso que erosiona su margen de actuación y limita sus decisiones en función de la lógica de los mercados. Este escenario se cierra con una extremada asimetría en la distribución de poder político y militar a escala mundial, que consagra la hegemonía de una única superpotencia que en ocasiones se resiste a la introducción de mecanismos institucionales del ámbito mundial, que podrían incidir positivamente en la regulación de los desajustes existentes, pero que supondrían una limitación de su autonomía (Protocolo de Kyoto, Tribunal Penal Internacional, etcétera).

En este sentido, se requieren cambios en el orden internacional, tanto en el plano económico como en el plano político-institucional, a fin de alcanzar una auténtica gobernabilidad democrática a escala global. Esta transformación pasa por un apoyo decidido, sincero y general a los procesos de democratización en los países atrasados, impulsado desde instancias multilaterales, pero requiere también de un nuevo diseño del marco de instituciones internacionales a fin de hacerlas más democráticas y operativas frente a los problemas asociados a la globalización.

\section{Conclusión}

Las políticas de desarrollo nacionales pueden actuar sobre los círculos viciosos internos —económicos, políticos y sociales - que afectan a los países hipodesarrollados. No obstante, hay que tener presente que las dinámicas sociales nacionales están fuertemente condicionadas hoy por hoy por la inserción en el proceso de globalización. A este respecto, coexisten otras visiones sobre el efecto final que, en el marco del orden internacional actual, tienen los factores externos sobre los círculos viciosos internos en los países del hipodesarrollo. Así pues, la corriente central en economía, vinculada principalmente a la teoría neoliberal del desarrollo, defiende el mutuo beneficio que las naciones avanzadas y atrasadas obtienen de las relaciones económicas internacionales y propugna la liberalización externa de las relaciones comerciales y de los flujos financieros en los países del hipodesarrollo. Como resultado de estas políticas liberalizadoras se potenciaría el crecimiento mediante el comercio exterior y se faci- 
litaría la financiación de los procesos de inversión en los países más atrasados a partir del ahorro externo, lo que contribuiría a la ruptura de los círculos viciosos internos del hipodesarrollo. Desde esta perspectiva, el principal peligro para las naciones del hipodesarrollo sería su falta de integración en la economía internacional, su marginación en el proceso de globalización. Por el contrario, el éxito económico de las nuevas naciones industrializadas del sureste asiático o China a lo largo de las últimas décadas hablarían de los efectos positivos de una apertura internacional controlada.

No obstante, las corrientes heterodoxas (economía marxista, teoría de la dependencia, estructuralismo, etcétera) adoptan una visión crítica de la repercusión que el orden internacional actual tiene sobre los países más desfavorecidos. Desde estos enfoques, se considera que las pautas actuales de inserción de las naciones hipodesarrolladas en la economía internacional contribuyen a la retroalimentación de su dinámica interna de empobrecimiento, dando lugar a un círculo vicioso internacional de la pobreza, que estaría intensificándose como consecuencia del modelo actual de globalización. La evolución reciente de algunos países del África Subsahariana podría representar un ejemplo ilustrativo de ello. Por consiguiente, desde esta perspectiva, no bastaría con las políticas de reforma en el ámbito interno para romper la dinámica perversa del hipodesarrollo, sino que la anulación de los círculos viciosos de la pobreza requeriría también de esfuerzos orientados a la transformación del orden internacional.

En cualquier caso, el desarrollo debe entenderse como una meta que implica crecimiento económico, gobernabilidad democrática y bienestar social con equidad: estos tres vértices representan objetivos parciales de desarrollo en una triple dirección de progreso económico, progreso político y progreso social. No obstante, no en pocas ocasiones, se ha defendido la incompatibilidad de estos objetivos, abogando por priorizar la acumulación de capital y el crecimiento económico a costa de la represión temporal de los derechos civiles y políticos o de la asunción de elevados niveles de desigualdad social ${ }^{5}$. Pese a que en ciertos países esta estrategia ha acabado dando algunos frutos positivos, no existe ninguna garantía de que estos resultados sean generalizables. Y aún en las experiencias favorables, esta estrategia supone una postergación

5 Perry et al. (1997) señalan la existencia de un círculo vicioso en Latinoamérica en función del cual un crecimiento económico lento determina niveles altos de pobreza, al mismo tiempo que la alta incidencia de la pobreza condiciona un crecimiento lento. De igual manera, reflexionan sobre cómo convertir esos círculos viciosos en círculos virtuosos en los que el crecimiento económico y la reducción de la pobreza se refuercen mutuamente. 
de objetivos de desarrollo que empobrece los logros alcanzados al conllevar sacrificios en términos de libertad y cohesión social para una o varias generaciones, cuyas consecuencias pueden llegar a ser estructurales.

Frente a este planteamiento, se ha defendido en este trabajo la hipótesis de que, bajo ciertas condiciones, es posible conseguir dinámicas virtuosas acumulativas que hacen compatibles y complementarios el crecimiento económico, la gobernabilidad democrática y el desarrollo social. Desde esta perspectiva, cada uno de estos objetivos o fines del desarrollo representa también un medio para alcanzar los restantes, en la medida en que cada uno contribuye al logro de los demás. A nuestro juicio, este modelo de desarrollo equilibrado, que implica logros progresivos en los ámbitos económico, político y social, constituye la estrategia más efectiva, desde el punto de vista instrumental, y deseable, desde el punto de vista teleológico, en pos del objetivo de desarrollo.

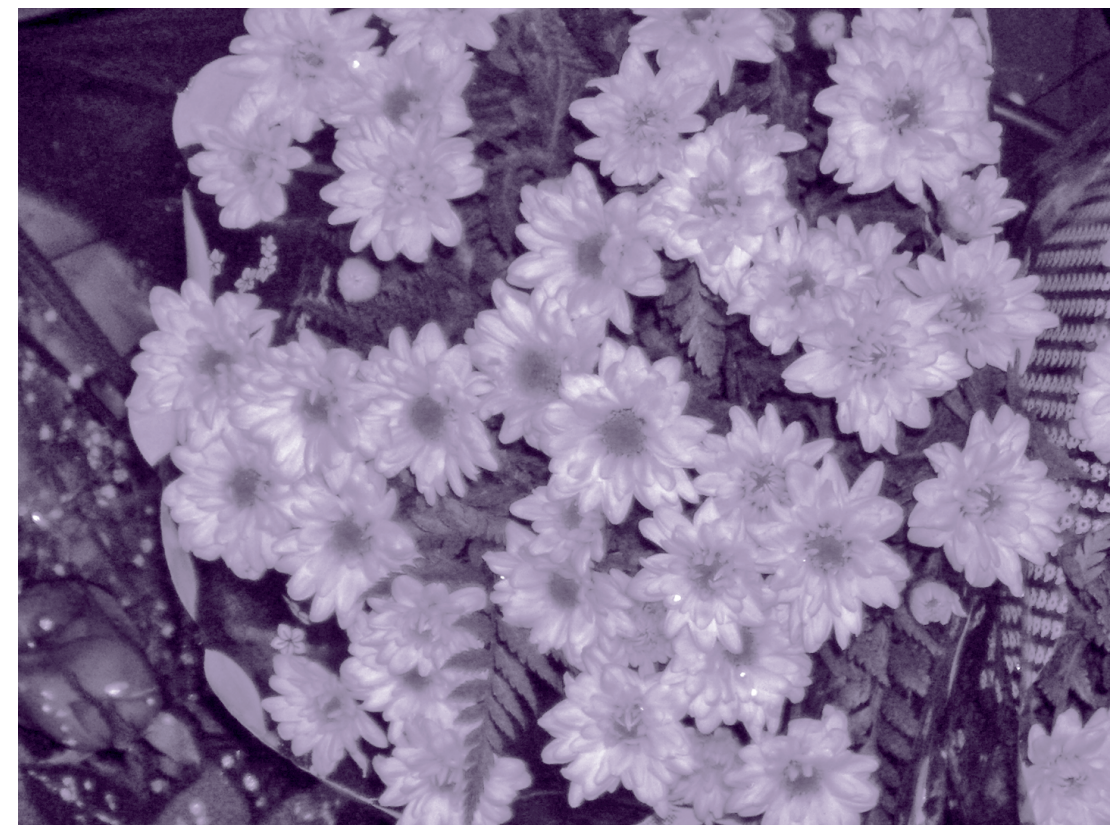

Buenos Aires, 2005. PAI 


\section{Bibliografía}

Alcaide, Laura y Larrú, José María, “Corrupción, ayuda al desarrollo, pobreza y desarrollo humano", Boletín Económico de Información Comercial Española, núm. 2917, Madrid, Ministerio de Industria, Turismo y Comercio , 2007, pp. 37-58.

Barro, Robert J., "Determinants of Democracy", Journal of Political Economy, vol. 107, Issue 6, Chicago, University of Chicago Press, 1999, pp. S158-S183. , "Democracy and Growth", Working Paper núm. w4909, Cambridge, NBER, 1994.

Dahl, Robert A., On Democracy, New Haven, Yale University Press, 1998.

Feng, Yi, "Democracy, Political Stability and Economic Growth", British Journal of Political Science, vol. 27, núm. 3, Cambridge, Cambridge University Press, 1997, pp. 391-418.

Fontela, Emilio y Guzmán, Joaquín, “Círculos viciosos y virtuosos del desarrollo económico", en Jorge Aromando (comp.), El desafío de la globalización en América Latina. Claves para una interpretación, Buenos Aires, Jorge Baudino Ediciones, 2006, pp. 91-120. , "La teoría circular del desarrollo. Un enfoque complejo", Estudios de Economía Aplicada, núm. 21-2, Almería, ASEPELT, 2003, pp. 221-242.

Helliwell, John F., "Empirical Linkages Between Democracy and Economic Growth", British Journal of Political Science, núm. 24, Cambridge, Cambridge University Press, 1994, pp. 225-248.

Kaufmann, Daniel y Kraay, Aart, "Growth Without Governance", World Bank Policy Research Working Paper, núm. 2928, Washington, DC, Banco Mundial, 2002. y Zodio-Lobatón, Pablo, "Governance Matters II-Updated Indicators 2001/02”, World Bank Policy Research Working Paper, núm. 2772, Washington, DC, Banco Mundial, 2002. y Mastruzzi, Massimo, "Governance Matters III: Governance Indicators for 1996-2002 Indicators for 2000/01", World Bank Policy Research Working Paper, Washington, DC, Banco Mundial, 2003.

Larrú, José María, "La ayuda para los pobres funciona: la eficacia micro de la ayuda al desarrollo", Revista de Economía Mundial, núm. 8, Huelva, Sociedad de Economía Mundial, 2003, pp. 97-117.

Myrdal, Gunnar, La pobreza de las naciones, Barcelona, Ariel, 1974.

Nurkse, Ragnar, Problemas de formación de capital en los países insuficientemente desarrollados, México, Fondo de Cultura Económica, 1955.

Perry, Guillermo E.; Arias, Omar S.; López, J. Humberto; Maloney, William F. y Servén, Luis, Poverty reduction and growth: Virtuous and vicious circles, Washington, DC, Banco Mundial, 2006.

Prats, Joan, "Gobernabilidad democrática para el desarrollo humano. Marco Conceptual y Analítico", Instituciones y Desarrollo, núm. 10, Barcelona, Instituto Internacional de Gobernabilidad de Cataluña, 2001.

PNUD, Informe sobre el desarrollo humano 2002. Profundizar la democracia en un mundo fragmentado, Nueva York, Ediciones Mundi-Prensa, 2002.

Rodrik, Dani, "Institutions for High-Quality Growth: What They Are and How to Acquire Them", Studies in Comparative International Development, vol. 35, núm. 3, Providence, Watson Institute for International Studies, 2000, pp. 3-31.

Sen, Amartya, Desarrollo y Libertad, Barcelona, Planeta, 1998.

Tavares, José y Wacziarg, Romain, "How democracy affects growth", European Economic Review, vol. 45, núm. 8, North-Holland, 2001, pp. 1341-1378. 